\title{
Merely Increasing Action Options Increases Charitable Donation
}

Erika Weisz ( $\nabla$ eweisz@fas.harvard.edu )

Harvard University

\section{Mina Cikara}

Harvard University https://orcid.org/0000-0002-6612-4474

\section{Article}

Keywords: altruism, donation, choice architecture, decision-making

Posted Date: August 28th, 2020

DOl: https://doi.org/10.21203/rs.3.rs-59021/v1

License: (9) This work is licensed under a Creative Commons Attribution 4.0 International License. Read Full License 


\section{Abstract}

Amid a sea of requests for aid, what factors affect decisions to donate? Researchers and practitioners continue to debate whether we ought to prioritize leveraging passion (e.g., appeal to people's empathy) or reason (e.g., appeal to utilitarian drives). Here we circumvent the challenges of emotions and obligation as levers by investigating the efficacy of a novel manipulation of choice architecture to affect giving: increasing the number of action options available to donors to increase how much they give. Across six experiments $(N=6,321)$, participants responded to single or multiple-bid donation requests. Viewing multiple bids for aid increased both intention to donate and actual donation (controlling for affective states that have been previously associated with costly helping). Notably, increasing the number of bids did not decrease the proportion of people who donated at all. We rule out previously-documented heuristics (i.e., $1 / n$, fairness) and agency as explanations for our effect, and find positive evidence for the role of merely increasing action options (i.e., bids). Finally, we replicate our effect in a natural experiment of charitable donations $(N=10,000)$ : presenting donors with multiple bids increased the average donation by $\$ 8.77$ (a $19.7 \%$ increase). Our findings have theoretical implications for invigorating behavior and offer practical suggestions for how charitable organizations can better engineer solicitations for aid.

\section{Introduction}

Charitable donation is a multi-billion dollar industry. In the US alone, people gave almost $\$ 428$ billion to non-profits, faith-based organizations, and other charities in 2018. Sixty-eight percent of these contributions came not from corporations or foundations, but rather from individual donors (Giving USA 2019: The Annual Report on Philanthropy for the Year 2018, 2019). Though these numbers are impressive, need reliably outpaces funding supply. How do we encourage people to give even more?

Researchers and practitioners continue to debate whether bids for aid ought to appeal to donors' passion (e.g., empathy) or reason (e.g., moral obligation), as both of these approaches are known to affect giving. However, the conditions under which specific strategies will increase (versus decrease) donations are not well defined. For instance, it is unclear when benefits garnered by evoking negative emotions (Hibbert et al., 2007; Small \& Verrochi, 2009) might outweigh those earned by evoking positive emotions (Ashar et al., 2016; Bekkers \& Wiepking, 2011; Genevsky \& Knutson, 2015; Park et al., 2019). And although appeal to individuals' moral obligation to maximize collective welfare can increase charitable donation (Bentham, 2015; Lindauer et al., 2020; Mill, 1970), this framing is not universally compelling: many people are more swayed by emotional appeals, even when the resulting donations do more harm than good (Bloom, 2017).

Research on the structure of donation bids circumvents the challenges of emotions and obligation as levers. Choice architecture-the strategic presentation of information and options in a choice set-shapes behavior across a wide variety of domains, ranging from organ donation (Johnson \& Goldstein, 2003) to purchasing goods and food consumption (Fox et al., 2005; Huber et al., 2016; Redden, 2008) to charitable giving (Oppenheimer \& Olivola, 2011). Here we investigate a novel manipulation of choice architecture to 
affect giving: whether increasing the number of action options available to donors increases donations, above and beyond gains driven by affect and perceived efficacy.

Choice architecture and motivation in donation. Several studies have manipulated donation bid features to increase giving to those in need (for a review, see Oppenheimer \& Olivola, 2011). For instance, some donation platforms allow donors to make single contributions that are subsequently divided among several causes, removing the need to allocate donations across multiple deserving recipients and causes. One advantage of this approach is that it reduces the possibility that would-be donors experience choice overload, which could ultimately reduce donations if people opt out altogether (Fasolo et al., 2007; lyengar \& Lepper, 2000). However, bundling aid requests in this manner could render fundraising campaigns less effective. For example, people donate more in aggregate when multiple people request aid because they want to be fair to all those in need (Sharps \& Schroeder, 2019; Soyer \& Hogarth, 2011)though only up to a point (Andreoni, 2007; Slovic, 2007). This effect is particularly pronounced when donors are asked to determine first how much they would give to one person, and then asked how much they want to give to a group of needy people (Hsee et al., 2013). Note, however, that these studies do not tell us anything about what happens when the donation targets are issues (e.g., raising funds for pandemic preparedness) rather than individuals, in which case fairness considerations are less relevant.

Why might increasing action options increase how much some people give? Though some have argued that agency or self-determination accounts for an initial increase in effort when people have more choices (Ryan \& Deci, 2000), we offer a different account in the context of giving, informed by research on motivation and response vigor. Once activated, motives can have two effects: a 'directing' effect which

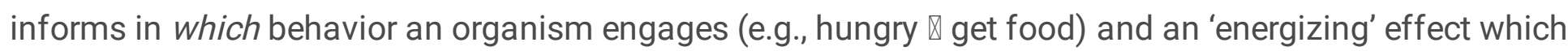
determines the vigor with which they behave (e.g., how much food to consume, Dickinson \& Balleine, 2002; Niv et al., 2006). In our experiments we first expose participants to a series of stories characterizing a crisis or cause, which may activate the motive to help among some but not all participants. We then give donors either a single action option (i.e., one bid) or multiple action options (i.e., multiple bids) by which to slake their motivation to help. If the motive to help is activated and people have selected an action ("see donation box $\otimes$ give") then they should give more when there are multiple action options available. This account makes a very specific prediction: donation should only increase with action options among those who are motivated to give. Said another way, we predict that increasing the number of actions available within a donation bid will not affect whether people donate, but conditional on deciding to donate, it will increase how much they give. This is important because it suggests that multiple bid donation requests should not backfire by reducing the number of people who donate at all.

Related but distinct phenomena. Pertinent to the current investigation are the phenomena of 'unpacking' and subadditivity. When people provide more detail about the constituent elements within a category it affects the way they think about that category as a whole (Rottenstreich \& Tversky, 1997; Van Boven \& Epley, 2003). For example, participants estimate that the likelihood of dying from unnatural causes is greater when this category is unpacked (as accidents, homicides, or other causes) relative to packed (as unnatural causes; Tversky \& Koehler, 1994). Indeed, the probability of the whole is often judged to be less 
than the summed probabilities of its parts, a phenomenon known as the subadditivity effect (Hilbert, 2012; Tversky \& Koehler, 1994). However, the unpacking and subadditivity findings refer specifically to the generation of probability estimates or extreme evaluations, not response vigor (or more specifically, donation behavior).

Of course, by increasing the number of action options (i.e., donation bids), one runs the risk of habituation to issue urgency; people may give less when there are multiple bids because each additional bid feels less pressing. Partition dependence-another related phenomenon whereby decisions vary systematically based on how options within a choice set are 'clustered' in decision-makers' minds (Fox et al., 2005; Johnson et al., 2012)-could potentially be used to counteract the effects of habituation (Redden, 2008). For example, instead of thinking of all issues as belonging to the superordinate category 'problems in the world' they could be portioned into "humanitarian" and "environmental" crises. If multiple bids for aid elicit an effect of habituation or diminished sensitivity to need, partition dependence could provide a remedy for this problem. Note, however, that a partition dependence account predicts equal allocation across partitions (the 1/n heuristic, Benartzi \& Thaler, 2001; or maximum entropy heuristic, Fox et al. 2005; Langer \& Fox, 2004), which makes a similar prediction as the fairness motive in charitable giving observed in other studies (Sharps \& Schroeder, 2019; Soyer \& Hogarth, 2011). Note also that partition dependence makes predictions about how money would be distributed, but not how much. Nevertheless, if the $1 / \mathrm{n}$ heuristic accounts for our effects, we should see equal allocation across actions options (i.e., even splits among self and issue bids). If a fairness explanation accounts for our effects, we should see equal allocation across issues or partitions.

Overview of the present research and hypotheses. We investigated whether (1) presenting multiple-action bids (as compared to single-action bids) and (2) partitioning bids into distinct categories (demarcated by labels) increased the amount of money participants donated to charitable causes. We also tested whether these effects could be accounted for by a $1 / n$ heuristic or fairness account, and whether they persisted after controlling for emotions like distress, empathy, and a sense of efficacy.

In Experiments $1 \mathrm{a}$ and $1 \mathrm{~b}$, participants viewed donation requests containing either single or multiple donation boxes, and partitioned or not, and indicated how they would hypothetically donate. In Experiment 2, participants viewed single or multiple donation boxes, partitioned or not, then made real donations from a bonus with which we endowed them. In Experiment 3, we manipulated the order of options within the bid to test whether our preceding results could be explained by differential salience of self-interest. In Experiment 4, we ruled out donors' agency as an explanation for our preceding results. In Experiment 5 we tested whether our results could be accounted for by a mere increase in action options. Note that across all conditions and experiments $(N=6,321)$, we instructed participants to indicate how much they wanted to keep to ensure self-interest was equally salient across all conditions. Finally, Experiment 6 tested the effect of multiple donation boxes in a natural experiment $(N=10,000)$ of real donations.

Across these experiments, we hypothesized that: 
H1: Multiple-bid requests (as compared to single-bid requests) would increase the overall amount donated to charitable causes controlling for self-reported affect;

H2: Partitioned donation requests (as compared to non-partitioned donation requests)

would increase the overall amount donated to charitable causes controlling for self-reported affect;

H3: Bids would interact with partitioning to increase the overall amount donated to charitable causes above and beyond two main effects, controlling for affect.

Open Science Statement. All materials, data, and analysis scripts can be found on OSF:

https://osf.io/tra86/?view_only=a73d349a2ee34ef894b55f8cf50041e6

\section{Experiment Section}

\section{Experiment 1a}

Experiment 1 a examined donation preferences as a function of donation structure (i.e., single or multiple bids for aid and the presence or absence of partitioning labels) and self-reported affect. Participants read article segments describing humanitarian and environmental issues and then indicated their emotional reactions to these issues, as well as their willingness to donate to causes addressing these issues. Sample size in the present experiments was based on an anticipated small effect size $(f=.10)$. Power analyses revealed that, in order to detect effects of this size with $80 \%$ power, each experiment with four groups required a minimum sample of 324 per cell. We therefore aimed to recruit 1096 participants for Experiments $1 \mathrm{a}, 1 \mathrm{~b}, 2$ and 3.

\section{Method}

Participants. The final sample consisted of 1103 participants (53.6\% female; age: $M=36.25, S D=11.79$ ) who were recruited through Amazon Mechanical Turk (MTurk), see Supplemental Materials for demographic information. There were no exclusions; all participants were included in analysis. In all experiments, participants had to complete a bot-screening question before starting. For all experiments, informed consent was obtained from all participants and procedures were approved by the university's Institutional Review Board.

Procedure. Participants were randomly assigned to one of four conditions in a 2 (donation request: single vs. multiple bids) $\times 2$ (issue partitioning: category labeled vs. unlabeled) between-subjects design. We informed all participants that they would read two article segments describing current, urgent issues; rate their feelings about the issues; then indicate how much money they would want to donate to charities addressing the issues. To encourage participants to engage with the material, we asked them to check a box pledging to read the articles carefully. Participants then read two article segments-one humanitarian and one environmental article segment-presented in random order (see Supplemental Materials for article segments). Each segment was randomly selected from a group of five humanitarian and five 
environmental issues; we included multiple scenarios in each experiment to assess the robustness of our effects and modelled scenario as a random effect.

For approximately half of the participants, headers above article segments contained a partition label indicating that the cause belonged to the category 'humanitarian' or 'environmental' (e.g., "Humanitarian Issue: Famine"). The other participants saw descriptive headers without partition labels (e.g., just saw "Famine"). After reading the two article segments, participants were asked to make ratings on five dimensions, all on a scale from 0 (not at all) to 100 (extremely). Specifically, participants made global ratings to indicate how distressed, compassionate, efficacious, and motivated they felt after reading about both issues, and how important they thought the issues were. These five measures were presented in a random order (see Supplemental Materials for question phrasing).

Donation request: Participants were then randomly assigned to view one of four donation requests (see Figure 1): a single vs. multiple bid request, with/without issue category labels. They were asked to imagine that they had fifty cents to allocate between charities and themselves.

Across all four conditions, the total amount of money on the two or three slider scales had to sum to fifty cents. Participants were not allowed to proceed until they met this constraint. Again, we included the 'keep' slider across all conditions to make sure participants were thinking in every case about how much they were giving up by donating. If there were no effect of donation bid structure on donations, the amount that participants kept should be the same across all four conditions.

\section{Results}

We conducted a linear mixed model followed by a two-way, between-subjects analysis of variance to examine whether the amount of money participants would hypothetically donate was affected by (1) the structure of the donation question-single or multiple bids-and (2) partitioned category labels. Models included two random effects (one for the humanitarian and one for the environmental item because each issue was randomly drawn from a set of 5), and were implemented using the Ime4 (Bates et al., 2015), ImerTest (Kuznetsova et al., 2017) and MuMln packages (Bartón, 2017) in R.

We observed a statistically significant main effect for multiple bid donation question structure, $F(1$, $1098.07)=37.85, p<.001$, see Figure S1A. On average, participants in the multiple bid conditions $(M=$ $35.00 \dot{c}, S D=18.42 \zeta)$ gave nearly $\$ 0.07$ more than participants in the single bid conditions $(M=28.08 \dot{c}$, $S D=19.15 \zeta), t(1101.00)=6.11, p<.001,95 \% \mathrm{Cl}[4.70,9.14], d=.37$. Contrary to our hypothesis, we observed a significant difference in the proportion of participants who donated in the multiple bid condition (89\%) versus the single bid condition (83\%), $\chi^{2}(1, N=1103)=7.17, p<0.01$. Spoiler: this difference was not statistically significant in any of the other experiments.

There was no main effect for partition category labels, $F(1,1096.52)=1.04, p=.31$, nor was the interaction of these two effects significant, $F(1,1087.30)=2.40, p=.12$, see Supplemental Materials for pairwise comparisons. 
We also tested whether the effect of multiple bids on total donation amount persisted after accounting for participants' self-reported affect. We centered the self-reported ratings of distress, compassion, efficacy, importance, and motivation, then entered them along with dummy-coded bid number and partition predictors, and random effects of issue, to predict total amount donated. Motivation, issue importance, and compassion were statistically significant predictors of the amount of money participants would be willing to donate, but the multiple bid manipulation remained significant (see Table S1A).

We next examined whether these effects could be accounted for by a $1 / n$ heuristic account by examining the proportion of participants who split donations evenly among the number of boxes on the page (25c per box in the single bid condition, 16-17 $\bar{c}$ per box in the multiple bid condition). Because $50 c$ is not evenly divisible by 3 , here we consider participants in the multiple bid (i.e., 3 box) condition who allocated $16 \phi, 17 \phi$ and $17 \phi$ in any configuration the three boxes as donating in line with a $1 / n$ heuristic. Of participants in the single bid condition, only $14.5 \%$ allocated $25 c$ per box. Not a single participant in the multiple bid condition gave any configuration of $16 \dot{c}, 17 \dot{c}$ and $17 \dot{\phi}$, as would have been predicted by a $1 / n$ heuristic account.

Finally, we examined whether donations in the multiple bid conditions were driven by concerns about fairness: that is, equal distribution of donations across the two issues (irrespective of how much they kept). Of participants who gave anything, only $24.1 \%$ donated the same amount to each issue. Nor did one issue dominate across participants; on average people allocated $17.44(\mathrm{SD}=14.06)$ cents to humanitarian issues and $17.56(S D=13.59)$ cents to environmental issues $t(1102.00)=-.15, p=.88,95 \%$ $\mathrm{Cl}[-1.76,1.51], d=-.01$.

In sum, even when controlling for variables related to affect, viewing an donation request with multiple bids (i.e., a bid for each issue) significantly increased the amount of money participants said they would donate. (This pattern was not driven by a $1 / n$ heuristic or equal distribution of donations across issues.) By contrast, partitioning did not significantly increase hypothetical donation amount.

However, it is possible that categorization was no longer salient at the time of donation. That is, without reinforcing the categories prior to donation, they became less conceptually distinct by the time participants were asked how much money they would want to donate. To address this possibility, in Experiment $1 \mathrm{~b}$ we partitioned articles into categories both at time of encoding and right before donation question. The detailed results of Experiment $1 \mathrm{~b}$ appear in the Supplemental Materials; but, in short, they replicated Experiment 1a (see Figure S1B). Again, there was only a significant main effect of the multiple bid manipulation (even when controlling for self-reported affect; see Table S1B).

\section{Experiment 2}

Experiments $1 \mathrm{a}$ and $1 \mathrm{~b}$ indicated that donation amounts varied as a function of the number of bids in a donation request, but these experiments were limited in that they examined hypothetical donations. In 
Experiment 2, participants completed a task almost identical to Experiment 1a, but were given the chance to make real donations from $\$ 0.50$ bonuses with which they were endowed.

\section{Method}

Participants. The final sample consisted of 1106 participants ( $57.8 \%$ female; age: $M=36.80, S D=12.14$ ) who were recruited through Amazon Mechanical Turk. All responses were included in analysis.

Procedure. The experimental procedure was the same as that described in Experiment 1a, with two exceptions. First, participants were endowed with a real $\$ 0.50$ bonus in addition to their base pay (rather than asking participants to imagine a hypothetical bonus). Second, we removed one environmental and one humanitarian article from the stimulus set because there were no university-approved charities addressing the issues described in those two articles (yielding four possible issues per issue category instead of five). As in Experiments $1 \mathrm{a}$ and $1 \mathrm{~b}$, participants were randomly assigned to one of four conditions: an Multiple-Labeled condition ( $n=276)$, a Single-Labeled condition ( $n=274)$, an MultipleUnlabeled condition $(n=277)$ and a Single-Unlabeled condition $(n=279)$.

\section{Results}

We analyzed the data in exactly the same way as Experiments $1 \mathrm{a}$ and $1 \mathrm{~b}$. Replicating $1 \mathrm{a}$ and $1 \mathrm{~b}$, we observed a statistically significant main effect for multiple bid donation question structure, $F(1,1101.06)$ $=10.76, p=.001$, see Figure 2. On average, participants in the multiple bid conditions $(M=29.66 \mathrm{c}, S D=$ $20.61 \dot{c})$ gave almost $\$ 0.04$ more than participants in the single bid conditions $(M=25.86 \dot{c}, S D=20.40 \dot{c})$, $t(1104.00)=3.09, p=.002,95 \% \mathrm{Cl}[1.39,6.23], d=.19$. Consistent with our hypotheses, we did not observe a significant difference in the proportion of participants who donated in the multiple bid condition (78\%) versus the single bid condition $(75 \%), \chi^{2}(1, N=1106)=1.14, p=0.29$.

As in Experiments $1 \mathrm{a}$ and $1 \mathrm{~b}$, there was no main effect for partition category labels, $F(1,1099.65)=$ $0.002, p=.96$, nor was the interaction of these effects significant, $F(1,1098.89)=0.38, p=.54$, , see Supplemental Materials for pairwise comparisons.

We next tested whether the effect of multiple bids remained significant after accounting for participants' self-reported affect. As in Experiments $1 \mathrm{a}$ and 1b, issue importance and compassion were statistically significant predictors of the amount of money participants donated, but the multiple bid manipulation remained significant (see Table S2). Distress also emerged as a significant predictor of donation, as did motivation (however this latter relationship was only marginally significant).

We also examined whether these effects could be accounted for by a $1 / n$ heuristic. Of participants in the single bid condition, $17.2 \%$ allocated $25 \mathrm{c}$ per box. Only one participant in the multiple bid condition $(0.18 \%)$ gave a configuration of $16 \dot{c}, 17 \dot{c}$ and $17 \dot{c}$.

Finally, we examined whether donations in the multiple bid conditions were driven by equal distribution across the two issues (i.e., fairness): only $29.3 \%$ of participants donated the same amount to each issue. 
In contrast to Experiments $1 \mathrm{a}$ and $1 \mathrm{~b}$, people gave more to humanitarian issues: on average people gave $16.02 \zeta(S D=14.63 \dot{c})$ to humanitarian issues and $13.65 \zeta(S D=13.22 \zeta)$ to environmental issues, $t(1104.00)=2.83, p=.005,95 \% \mathrm{Cl}[.73,4.02], d=.17$

In sum, we replicated the effect of multiple bids with real donations and confirmed that multiple bids did not decrease the proportion of people who opted to donate at all. Consistent with our predictions, increasing action options did not affect people's decision whether to give, only how much.

\section{Experiment 3}

In Experiments 1a, 1b, and 2, we found evidence that multiple bids for aid garnered greater donations than single bids even though we asked participants across all conditions to be explicit in how much they wanted to keep. However, in the preceding experiments participants were asked how much they wished to keep only after they had already said how much they wanted to donate to each bid. Note that participants could always go back and edit the donation sliders, but it is possible that once they were anchored on their donations they would be reluctant to change them. One possible consequence of this is that selfinterest was not equally salient in multiple bids versus single bid donations requests. Experiment 3 tests the possibility that the effect of multiple bids is driven by the ordering of these questions.

\section{Method}

Experiment 3 was similar to that of Experiment 1a, with two exceptions. First, because Experiments 1a, $1 \mathrm{~b}$, and 2 revealed no effect of partitioning as compared to control, we removed this manipulation. Instead, we showed all participants the partitioned stimuli (i.e., those with category labels). Second, we randomized whether the "keep" slider was the first item or the last item on the donation request page.

Participants. The full sample consisted of 1105 participants recruited through Amazon Mechanical Turk. 15 responses were excluded from analysis: 14 from participants who had already completed a previous experiment in this set, and 1 from a participant who completed the present experiment twice (their first response is included in this dataset), leaving a final sample of $N=1090$ (56.9\% female; age: $M=36.80$, $S D=11.53)$.

Procedure. Participants read two article segments describing current issues (one issue per category, randomly selected from the same sets used in 1 and $1 \mathrm{~b}$ ) and rated their affect, identical to the previous experiments. Participants were then randomly assigned to view one of four donation requests: a Single-'Keep' Last condition ( $\mathrm{N}=274$ ), where the "keep" slider came last after a bundled humanitarian and environmental slider (identical to the "Single-Labeled" condition in Experiment 1a), a Multiple-'Keep' Last condition ( $\mathrm{N}=273$ ), where the "keep" slider came last after a humanitarian slider and an environmental slider (identical to the "Multiple-Labeled" condition in Experiment 1a), a Single-'Keep' First condition ( $\mathrm{N}=270$ ) where the "keep" slider came first (followed by a bundled humanitarian and environmental slider), and an Multiple-'Keep' First condition ( $N=273)$, where the "keep" scale came first 
(followed by a slider for donation to the humanitarian issue and a slider for donation to the environmental issue). As in Experiments $1 a$ and $1 b$, the donation question was hypothetical; participants were asked to imagine that they have fifty cents to allocate between themselves and the charities addressing the issues about which they had just read.

\section{Results}

We conducted a linear mixed model followed by a two-way, between-subjects analysis of variance to examine whether the amount of money participants would hypothetically donate was affected by (1) the structure of the donation question-single or multiple bids-and (2) whether the "keep" slider came first or last. Models included two random effects: one for the humanitarian and one for the environmental item (because each issue was randomly drawn from a set of 5).

Replicating all of our previous experiments, we observed a main effect of multiple bids $F(1,1086.00)=$ $21.31, p<.001$. On average, participants in the multiple bids conditions $(M=33.24 \dot{c}, S D=19.05 \dot{c})$ gave approximately $\$ 0.05$ more than participants in the single bids conditions $(M=27.95 \mathrm{c}, S D=18.90 \mathrm{c})$, $t(1088.00)=4.60, p<.001,95 \% \mathrm{Cl}[3.03,7.54], d=.28$. In other words, the effect of multiple bids persisted even when participants decided first how much they wanted to keep, see Figure S2. Consistent with our hypotheses, we did not observe a significant difference in the proportion of participants who donated in the multiple bid condition (84\%) versus the single bid condition $(82 \%), \chi^{2}(1, N=1090)=.36, p=0.55$.

We also observed a main effect for the positioning of the keep slider, $F(1,1086.00)=7.54, p=.006$; participants who made decisions about how much to donate first $(M=32.16 \dot{\zeta}, S D=18.71 \zeta)$ gave approximately $\$ 0.03$ more than participants who decided how much they wanted to keep before making donations $(M=29.03 \dot{c}, S D=19.47 \dot{\zeta}), t(1088.00)=2.71, p=.007,95 \% \mathrm{Cl}[.86,5.40], d=.16$. Finally, the interaction of these two effects was not significant, $F(1,1086.00)=0.13, p=.72$, see Supplemental Materials for pairwise comparisons.

We next tested whether the effect of multiple bids remained after accounting for participants' selfreported affect. Issue importance, compassion, motivation, and distress all emerged as statistically significant predictors of the amount of money participants donated, but both the multiple bids manipulation and the keep position manipulation remained significant (see Table S3).

We also examined whether these effects could be accounted for by a $1 / n$ heuristic. Of participants in the single bid condition, $17.1 \%$ allocated $25 \mathrm{c}$ per box. Only three participants in the multiple bid condition $(0.55 \%)$ gave a configuration of $16 \dot{c}, 17 \dot{\phi}$ and $17 \dot{c}$.

Finally, we examined whether participants distributed donations equally across the two issues in the multiple bid conditions: only $24.9 \%$ of participants donated the same amount to each issue. On average people allocated $16.94(S D=14.30)$ cents to humanitarian issues and $16.29(S D=13.65)$ cents to environmental issues, $t(1090.00)=.76, p=.44,95 \% \mathrm{Cl}[-1.01,2.31], d=.05$. 
In sum, Experiment 3 replicated the previous three experiments and ruled out the possibility that that the multiple bids effect was driven entirely by the order in which people made donation decisions.

\section{Experiment 4}

In all previous experiments, participants who saw donation requests with multiple bids were able to allocate how much money they wanted to donate to each charity. However, participants who saw singlebid donation requests were not able to choose how to divide their donation. Thus one possible explanation is that participants who saw multiple bids had more agency-control over the manner in which their donations were distributed-and were therefore motivated to give more (as predicted by selfdetermination theory; Zuckerman, Porac, Lathin, \& Deci, 1978). If this is the case, then giving people in the single bid conditions an opportunity to specify how their single donation is allocated should make their total donations more similar to participants in the multiple bid condition than the single bid condition.

\section{Method}

Participants. Power analyses indicated that, in order to detect effects of this size with $80 \%$ power, each experiment with three groups required a minimum sample of 323 per cell. We therefore aimed to recruit 969 participants for Experiments 4 and 5. In Experiment 4, the sample consisted of 969 participants recruited through Amazon Mechanical Turk. 9 responses were excluded from analysis from participants who had already completed a previous experiment in this set, leaving a final sample of $\mathrm{N}=960(55.2 \%$ female; age: $M=35.81, S D=11.52$ ).

Procedure. Participants read two article segments describing current issues (one issue per category, randomly selected from the same sets used in 1, 1b, and 3) and reported affect, as in the previous experiments. Participants were then randomly assigned to view one of three donation requests: an Multiple-Labeled condition ( $N=319$, identical to the "Multiple-Labeled" condition in Experiment 1a), a Single-Labeled condition ( $N=320$, identical to the "Single-Labeled" condition in Experiment 1a), and a Single-Agency condition ( $N=321$ ), where participants indicated how they would want to allocate their total donation between the two issues prior to specifying their single donation (see Figure S3). The donation question was again hypothetical; participants were asked to imagine that they had fifty cents to distribute to donations and/or keep.

\section{Results}

To examine whether condition affected the amount of money participants would hypothetically donate, we conducted a linear mixed model (to account for random effects: one for the humanitarian and one for the environmental item) followed by one-way analysis of variance test. We observed a significant overall effect of condition, $F(2,956.75)=9.86, p<.001$. Replicating all our previous findings, pairwise comparisons using the emmeans package in $\mathrm{R}$ (Lenth, 2019) indicated that participants in the MultipleLabeled condition $(M=34.93 \dot{c}, S D=18.70 \hat{c})$ said that they would donate significantly more than 
participants in the Single-Labeled condition $(M=29.30 \mathrm{C}, S D=18.15 \mathrm{c}), t(953.12)=3.93, p<.001,95 \% \mathrm{Cl}$ $[2.27,8.98]$ and the Single-Agency condition $(M=29.60 \dot{c}, S D=17.27 \zeta), t(956.58)=3.74, p<.001,95 \% \mathrm{Cl}$ $[1.99,8.71]$. Participants in the Single-Agency condition indicated that they would donate more than participants in the Single-Labeled condition, but this difference was not significant, $t(953.53)=.19, p=$ $.98,95 \% \mathrm{Cl}[-3.10,3.64]$, see Figure S4. Consistent with our hypotheses, we did not observe a significant difference in the proportion of participants who donated in the Multiple-Labeled condition (87\%) versus the Single-Labeled condition (88\%) or the Single Agency condition $(87 \%), \chi^{2}(2, N=960)=.17, p=0.92$.

As in previous experiments, we examined whether these effects could be accounted for by a $1 / n$ heuristic. Only $15 \%$ of participants in the Single-Labeled condition and $20.1 \%$ of participants in the Single-Agency condition allocated 25 $\dot{c}$ per box. Not a single participant in the Multiple-Labeled condition gave a configuration of $16 \dot{c}, 17 \dot{c}$ and $17 \dot{\phi}$.

We next examined whether participants distributed donations equally across the two issues in the Multiple-Label condition as predicted by a fairness account: only $26.0 \%$ of participants donated the same amount to each issue. On average people allocated $17.97 \zeta(S D=13.63 \hat{c})$ to humanitarian issues and $16.96 \dot{C}(\mathrm{SD}=13.15 \dot{\zeta})$ to environmental issues, $t(636.00)=.96, p=.34,95 \% \mathrm{Cl}[-1.06,3.10], d=.08$.

Finally, participants in the Single-Agency condition demonstrated a stronger preference for equal distribution than their counterparts in the Multiple-Labeled condition in this and previous experiments (see Table S4).

Experiment 4 indicated that agency could not account for the effect of multiple bids we observed in real and hypothetical donations in the preceding experiments. Even when participants who viewed single-bid requests were able to indicate how they wanted to allocate their donations to different charities, they still said they would donate less than their counterparts who viewed requests with multiple bids.

\section{Experiment 5}

Experiment 5 tests whether merely increasing the number of action options-even in the absence of meaningful action labels-increases donations.

\section{Method}

Participants. The full sample consisted of 965 participants recruited through Amazon Mechanical Turk. 9 responses were excluded from analysis: 4 from participants who had already completed a previous experiment in this set, and 5 from participants who completed the present experiment multiple times (their first responses are included in the present dataset), leaving a final sample of $\mathrm{N}=956(49.6 \%$ female; age: $M=37.35, S D=11.86$ ).

Procedure. As in all previous experiments, participants read two article segments describing current issues (randomly selected from the same set used in Experiments 1a, 1b, 3 and 4) and reported their 
affect. Participants were randomly assigned to view one of three donation requests: a Multiple-Labeled condition ( $\mathrm{N}=319$, identical to the "Multiple-Labeled" condition in Experiment 1a), a Single-Unlabeled condition ( $\mathrm{N}=317$, identical to the Single-Unlabeled" condition in Experiment 1a), and a MultipleUnspecified condition ( $\mathrm{N}=320)$, where participants were asked to indicate how much money they would want to donate to charities on separate slider scales, but were not provided with any further information (see Figure S5). As in Experiments 1a, 1b, 3, and 4 the donation question was hypothetical; participants were asked to imagine that they have fifty cents to donate, or to keep for themselves.

\section{Results}

To examine whether condition affected the amount of money participants would hypothetically donate, we conducted a linear mixed model followed by a one-way analysis of variance test which accounted for two random effects (one for the humanitarian and one for the environmental item). We observed a significant overall effect of condition, $F(2,953.00)=17.36, p<.001$. Pairwise comparisons using the emmeans package in $\mathrm{R}$ (Lenth, 2019) revealed that participants in the Multiple-Labeled condition ( $M=$ $33.45 c, S D=18.08 c)$ indicated that they would donate significantly more than participants in the SingleUnlabeled condition $(M=26.00 \dot{c}, S D=17.17 \dot{c}), t(945.90)=5.19, p<.001,95 \% \mathrm{Cl}[4.08,10.82]$. Participants in the Multiple-Unspecified condition $(M=33.09 \phi, S D=18.64 \zeta)$ also indicated that they would donate significantly more than participants in the Single-Unlabeled condition, $t(949.77)=4.95, p<$ $.001,95 \% \mathrm{Cl}[3.73,10.46]$. Participants in the Multiple-Labeled condition indicated that they would donate more than participants in the Multiple-Unspecified condition, but this difference was not significant, $t(952.62)=.25, p=.97,95 \% \mathrm{Cl}[-3.00,3.71]$, see Figure S6. Consistent with our hypotheses, we did not observe a significant difference in the proportion of participants who donated in the Multiple-Labeled condition (87\%) versus the Multiple-Unspecified condition (85\%) or the Single Agency condition (86\%), $\chi^{2}(2, N=956)=.46, p=0.79$.

As in previous experiments, we examined whether these effects could be accounted for by a $1 / n$ heuristic. Only $15.8 \%$ of participants in the Single-Unlabeled condition allocated 25 ç per box. Two participants in the Multiple-Labeled (0.63\%) and no participants in the Multiple-Unspecified condition gave a configuration of $16 \dot{c}, 17 \dot{c}$ and $17 \dot{c}$.

Next we examined whether participants distributed donations equally across the two issues in the Multiple-Label condition as predicted by the fairness account: only $22.9 \%$ of participants donated the same amount to each issue. On average people allocated 16.91 (SD = 12.83) cents to humanitarian issues and $16.54(\mathrm{SD}=12.47)$ cents to environmental issues, $t(636.00)=.38, p=.71,95 \% \mathrm{Cl}[-1.59$, 2.34], $d=.03$.

Finally, we examined whether participants distributed donations equally across the two bids in the Multiple-Unspecified condition: $33.8 \%$ of participants donated the same amount to each issue. On average people allocated $15.77(S D=11.96)$ cents to the first bid and $17.33(S D=13.23)$ cents to the second bid, $t(638.00)=-1.56, p=.12,95 \% \mathrm{CI}[-3.51, .40], d=-.12$. 
In sum, merely increasing action options increased how much people donated.

\section{Experiment 6}

We analyzed data from 10,000 donors on an online donation platform to test whether multiple bids increased donations when (i) people were donating their own money, (ii) there were more than two action options, and (iii) the issues were of immediate and widespread public importance (e.g., current public health crises, social justice movements).

\section{Method}

We were provided with data by an online donation platform that randomly assigned donors to land on a contribution form that had a single bid (i.e., one donation box, Figure 3A) or multiple bids (i.e., between 2 and 21 donation boxes with different entities associated with each box; Figure 3B). In the Spring of 2020, over 10,000 donors landed on one of these two versions of the contribution page for a variety of public health and social justice issues (though the company declined to share for privacy purposes which issue corresponded to each donation). We received data for the first 5,000 respondents in each treatment. Donors could not see alternate variations if they closed out a form and soon after re-loaded the page. However, donors in the single bid condition had the option to expand the donation page to see the different recipient entities (effectively exposing them to the multiple bids condition). Therefore, we analyze the data first with the full sample and then again excluding anyone in the single bid condition who donated unequally across entities (indicating that they had expanded the donation page and deviated from the equal split allocations that were entered by default).

\section{Results}

In the full sample, participants in the multiple bids condition $(M=\$ 53.36, S D=85.35)$ gave significantly more than participants in the single bid condition $(M=\$ 44.54, S D=81.32), t(9974.67)=5.29, p<.001$, $95 \% \mathrm{Cl}[5.55,12.09], d=.11$; Figure $3 \mathrm{C}$. These results replicated in the restricted sample: single bid condition $(\mathrm{n}=4863 ; M=\$ 44.59, S D=81.35)$ versus complete multiple bids condition, $t(9856.97)=$ $5.23, p<.001,95 \% \mathrm{Cl}[5.49,12.07], d=.11$. In other words, donors in the multiple bids condition gave $\$ 8.77$ more on average (a $19.7 \%$ increase) than donors in the single bids condition.

This effect was not driven by outliers. The effect was present in a subset of donations equal to or below $\$ 100$, mean difference $=\$ 5.14, t(9417.96)=8.73, p<.001,95 \% \mathrm{Cl}[3.98,6.29], d=.18$, and equal to or below $\$ 10$, mean difference $=\$ 0.49, t(2155.83)=4.39, p<.001,95 \% \mathrm{Cl}[.27, .70], d=.18$ (Figure 3D-E).

Finally, we examined a model that included a main effect of number of action options (i.e., the number of entities on the form) among participants in the multiple bids condition to test whether the effect of multiple bids decreased as the number of action options increased (i.e., an action "overload" effect). Because there were 11 or fewer donors per cell who saw forms with greater than 11 action options, we subset the data to include only donors who saw forms with 10 or fewer action options. We found that- 
when examining all data in this subset-there was a positive main effect of action items, $b=10.14$ (95\% $\mathrm{Cl}[8.87,11.41]) ; t=15.68, p<.001$; donations increased as the number of boxes increased (up to 10). We observed the same effect for donations of $\$ 100$ or less, $b=2.93(95 \% \mathrm{Cl}[2.44,3.42]) ; t=11.71, p<.001$. However, when examining donations of $\$ 10$ or less within this subset, the effect was not significant, $b=$ $-.03(95 \% \mathrm{Cl}[-.16, .10]) ; t=-.43, p=.66$ (see Figure S7).

In sum, multiple bids (relative to single bid) donation forms significantly increased donation amounts when people were giving their own money to salient issues with widespread social and public health implications. Increasing the number of action options increased donations, except for the subset of donations at or under $\$ 10$. One possibility is that the number of action items on a page increases donations, but only when donations are relatively large. An alternative possibility is that we were not sufficiently powered to detect the effect in the $<\$ 10$ donation subset.

\section{Discussion}

Across seven experiments, we found that participants' hypothetical and actual donations to charity were higher when they saw donation requests with multiple bids, as compared to participants who saw a donation request with a single bid. This effect was not accounted for by previously documented heuristics (i.e., $1 / n$, fairness), differences in the salience of self-interest, or participants' sense of agency in allocation of donations. Nor did multiple bids decrease the proportion of individuals who donated across treatments. Instead, merely increasing action options-the number of bids-even when the options had arbitrary labels, was sufficient to increase total donation amounts relative to a single-bid request. These results replicated in a natural experiment of actual donations to public health crisis and social justice movement campaigns.

Although the simplicity of single-bid requests may be attractive to donors, our data indicate that multiplebid requests result in greater giving (again, without reducing the proportion of people who give at all). This observation is especially important in the age of online fundraising, where many donation platforms are organized around principles of least resistance (i.e., making it easy for donors to donate). Our findings indicate that fundraising campaigns could be even more effective if they present donors with multiple bids after bringing them to the donation page. In the natural experiment, presenting donors with multiple bids increased the average donation by $\$ 8.77$ (a $19.7 \%$ increase relative to the single bid condition). Of course, that figure is based on data obtained from a real-world, natural experiment where people sought opportunities to donate (i.e., the motive to give was already activated). In Experiment 2, participants were given the opportunity to donate (or keep) as much of an endowed bonus as they wished. Unlike donors in the natural experiment, participants in Experiment 2 had not specifically sought opportunities to give. Among these individuals, presenting multiple bids still increased donations by nearly $\$ 0.04$ out of $\$ 0.50$. Although this difference may seem small, it stands to have a powerful impact at scale. For example, if the 292 billion dollars given by individual donors in 2018 were made in response to single-bid requests, the 8 cents on the dollar gained from presenting multiple bids would result in an additional 23 billion given to charitable organizations around the world. 
Recent experiments have noted that choice models may be best characterized by an inverted U-shape, such that motivation (for instance, to buy a product) initially increases as the number of options increases (Grant \& Schwartz, 2011; Reutskaja et al., 2018; Shah \& Wolford, 2007) but that too many choices can lead to a decrease in motivation to make a decision (lyengar \& Lepper, 2000). Indeed, previous work demonstrates that benefits gained from increasing numbers of aid-seekers eventually asymptotes or even drops off completely (Sharps \& Schroeder, 2019; Slovic, 2007; Soyer \& Hogarth, 2011), perhaps due to donors' understanding of diminishing marginal aid to each additional victim (Andreoni, 2007). However, we find no evidence for this curvilinear pattern when the bids correspond to issues rather than people. We saw a positive effect of action options (up to 10) on donations over $\$ 10$. Future research will have to test the limits of this linear relationship.

Here we introduce a novel manipulation of choice architecture in the context of giving informed by research on motivation and behavioral invigoration: we found that increasing action options increased charitable giving. Specifically, we found that presenting multiple bids (as compared to single bids) increased the amount of money participants would donate, but not the proportion of participants who donated at all. In addition to offering practical suggestions for how charitable organizations might more effectively solicit aid, these findings also offer new insights into the relationship between motivation and response vigor more broadly.

\section{Declarations}

\section{Acknowledgements.}

This work was supported by a grant from the Pershing Square Fund for Research on the Foundations of Human Behavior awarded to E.W. and an NSF CAREER grant (BCS-1653188) awarded to MC.

\section{References}

Andreoni, J. (2007). Giving gifts to groups: How altruism depends on the number of recipients. Journal of Public Economics, 91(9), 1731-1749. https://doi.org/10.1016/j.jpubeco.2007.06.002

Ashar, Y. K., Andrews-Hanna, J. R., Yarkoni, T., Sills, J., Halifax, J., Dimidjian, S., \& Wager, T. D. (2016). Effects of compassion meditation on a psychological model of charitable donation. Emotion, 16(5), 691705. https://doi.org/10.1037/emo0000119

Bartón, K. (2017). MuMIn: Multi-model inference.

Bates, D., Maechler, M., Bolker, B., \& Walker, S. (2015). Fitting linear mixed-effects models using Ime4. Journal of Statistical Software, 67, 1-48. Journal of Statistical Software, 67(1-48).

Bekkers, R., \& Wiepking, P. (2011). A literature review of empirical studies of philanthropy: Eight mechanisms that drive charitable giving. In Nonprofit and Voluntary Sector Quarterly (Vol. 40, Issue 5). 
https://doi.org/10.1177/0899764010380927

Bentham, J. (2015). An introduction to the principles of morals and legislation. In Utilitarianism (pp. 7398). Doubleday.

Bloom, P. (2017). Against empathy: The case for rational compassion. Random House.

Dickinson, A., \& Balleine, B. (2002). The role of learning in the operation of motivational systems. Stevens' Handbook of Experimental Psychology.

Fasolo, B., McClelland, G. H., \& Todd, P. M. (2007). Escaping the tyranny of choice: When fewer attributes make choice easier. Marketing Theory, 7(1), 13-26. https://doi.org/10.1177/1470593107073842

Fox, C. R., Ratner, R. K., \& Lieb, D. S. (2005). How subjective grouping of options influences choice and allocation: Diversification bias and the phenomenon of partition dependence. Journal of Experimental Psychology: General, 134(4), 538-551. https://doi.org/10.1037/0096-3445.134.4.538

Genevsky, A., \& Knutson, B. (2015). Neural affective mechanisms predict market-level microlending. Psychological Science, 26(9), 1411-1422. https://doi.org/10.1177/0956797615588467

Giving USA 2019: The Annual Report on Philanthropy for the Year 2018. (2019).

Grant, A. M., \& Schwartz, B. (2011). Too much of a good thing: The challenge and opportunity of the inverted U. Perspectives on Psychological Science, 6(1), 61-76.

https://doi.org/10.1177/1745691610393523

Hibbert, S., Smith, A., Davies, A., \& Ireland, F. (2007). Guilt appeals: Persuasion knowledge and charitable giving. Psychology and Marketing, 24(8), 723-742. https://doi.org/10.1002/mar.200xx

Hilbert, M. (2012). Toward a synthesis of cognitive biases: How noisy information processing can bias human decision making. Psychological Bulletin, 138(2), 211-237. https://doi.org/10.1037/a0025940

Huber, J., Payne, J. W., Puto, C., Huber, J., Payne, J. W., \& Puto, C. (2016). Similarity Hypothesis Linked references are available on JSTOR for this article: Adding Asymmetrically Dominated Alternatives: Violations of Regularity and the Similarity Hypothesis. Journal of Consumer Research, 9(1), 90-98.

lyengar, S. S., \& Lepper, M. R. (2000). When choice is demotivating: Can one desire too much of a good thing? Journal of Personality and Social Psychology, 79(6), 995-1006. https://doi.org/10.1037/00223514.79.6.995

Johnson, E. J., \& Goldstein, D. (2003). Do Defaults Save Lives? Science, 302(5649), 1338-1339. https://doi.org/10.1126/science.1091721

Johnson, E. J., Shu, S. B., Dellaert, B. G. C., Fox, C. R., Goldstein, D. G., Häubl, G., Larrick, R. P., Payne, J. W., Peters, E., Schkade, D., Wansink, B., \& Weber, E. U. (2012). Beyond nudges: Tools of a choice architecture. 
Marketing Letters, 23(2), 487-504. https://doi.org/10.1007/s11002-012-9186-1

Kuznetsova, A., Brockhoff, P. B., \& Christensen, R. H. B. (2017). ImerTest Package: Tests in Linear Mixed Effects Models. Journal of Statistical Software, 82(13). https://doi.org/10.18637/jss.v082.i13

Lenth, R. (2019). emmeans: Estimated marginal means, aka least-squares means.

Lindauer, M., Mayorga, M., Greene, J., Slovic, P., Västfjäll, D., \& Singer, P. (2020). Comparing the effect of rational and emotional appeals on donation behavior. Judgment and Decision Making, 15(3), 413-420. https://orcid.org/0000-0002-3451-2966.

Mill, J. S. (1970). On liberty, utilitarianism, and other essays. Oxford University Press, USA.

Niv, Y., Joel, D., \& Dayan, P. (2006). A normative perspective on motivation. Trends in Cognitive Sciences, 10(8), 375-381. https://doi.org/10.1016/j.tics.2006.06.010

Oppenheimer, D. M., \& Olivola, C. Y. (2011). The science of giving: Experimental approaches to the study of charity. Psychology Press.

Park, B. K., Genevsky, A., Knutson, B., \& Tsai, J. (2019). Culturally Valued Facial Expressions Enhance Loan Request Success. Emotion. https://doi.org/10.1037/emo0000642

Redden, J. P. (2008). Reducing Satiation: The Role of Categorization Level. Journal of Consumer Research, 34(5), 624-634. https://doi.org/10.1086/521898

Reutskaja, E., Lindner, A., Nagel, R., Andersen, R. A., \& Camerer, C. F. (2018). Choice overload reduces neural signatures of choice set value in dorsal striatum and anterior cingulate cortex. Nature Human Behaviour, 2(12), 925-935. https://doi.org/10.1038/s41562-018-0440-2

Rottenstreich, Y., \& Tversky, A. (1997). Unpacking, Repacking, and Anchoring: Advances in Support Theory. Psychological Review, 104(2), 406-415. https://doi.org/10.1037/0033-295X.104.2.406

Shah, A. M., \& Wolford, G. (2007). Buying behavior as a function of parametric variation of number of choices: Short report. Psychological Science, 18(5), 369-370. https://doi.org/10.1111/j.14679280.2007.01906.x

Sharps, D. L., \& Schroeder, J. (2019). The Preference for Distributed Helping. Journal of Personality and Social Psychology, 117(5), 954-977. https://doi.org/10.1037/pspi0000179

Slovic, P. (2007). \&quot; If I look at the mass I will never act\&quot;:: Psychic numbing and genocide. Judgment and Decision Making, 2(2), 79-95. https://doi.org/10.1007/978-90-481-8647-1_3

Small, D. A., \& Verrochi, N. M. (2009). The face of need: Facial emotion expression on charity advertisements. Journal of Marketing Research, 46(6), 777-787. https://doi.org/10.1509/jmkr.46.6.777 
Soyer, E., \& Hogarth, R. M. (2011). The size and distribution of donations: Effects of number of recipients. Judgment and Decision Making, 6(7), 616-628.

Tversky, A., \& Koehler, D. J. (1994). Support theory: A nonextensional representation of subjective probability. Psychological Review, 101(4), 547-567. https://doi.org/10.1037/0033-295x.101.4.547

Van Boven, L., \& Epley, N. (2003). The unpacking effect in evaluative judgments: When the whole is less than the sum of its parts. Journal of Experimental Social Psychology, 39(3), 263-269.

https://doi.org/10.1016/S0022-1031(02)00516-4

Zuckerman, M., Porac, J., Lathin, D., \& Deci, E. L. (1978). On the Importance of Self-Determination for Intrinsically-Motivated Behavior. Personality and Social Psychology Bulletin, 4(3), 443-446. https://doi.org/10.1177/014616727800400317

\section{Figures}

\section{Single-Labeled}

Indicate below how much you would want to donate to humanitarian and environmental charities addressing these issues in cents, and how much you would want to keep. Note that the total amount must sum to $\mathbf{5 0}$ cents.

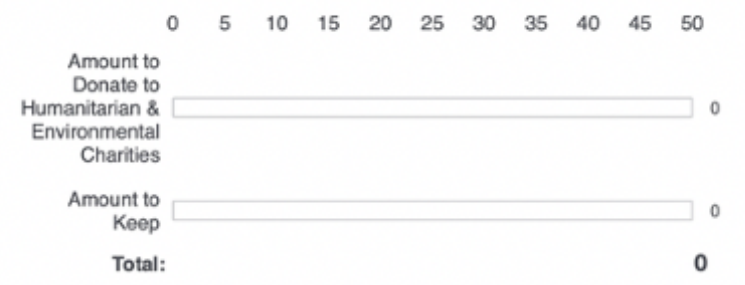

Single-Unlabeled

Indicate below how much you would want to donate to charities addressing these issues in cents, and how much you would want to keep. Note that the total amount must sum to 50 cents.

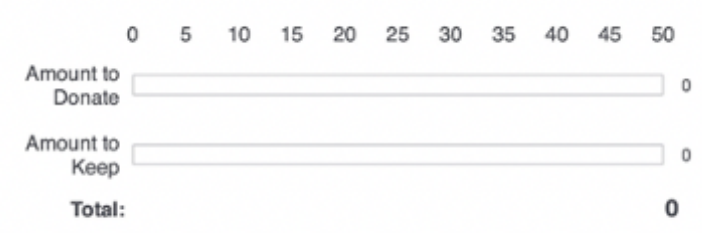

\section{Multiple-Labeled}

Indicate below how much you would want to donate to humanitarian and environmental charities addressing these issues in cents, and how much you would want to keep. Note that the total amount must sum to 50 cents.

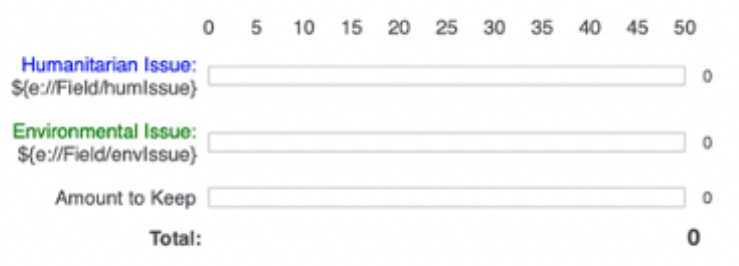

\section{Multiple-Unlabeled}

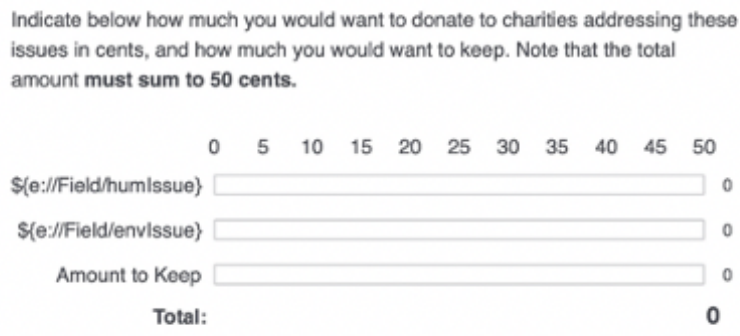

\section{Figure 1}

Experiment 1a ( $\mathrm{N}=1103)$ donation request pages: 2 (donation question: single vs. multiple bids) $\times 2$ (partitioning: category labeled vs. unlabeled) between-subjects design. Please note that the factor labels above the instructions did not appear in the experiment. To ensure self-interest was equally salient across all conditions, participants always had to indicate how much they wanted to keep. In multiple bid 
conditions, we piped the specific issue participants had read about on the previous pages (e.g., "Famine," "Climate change") into the slider label.

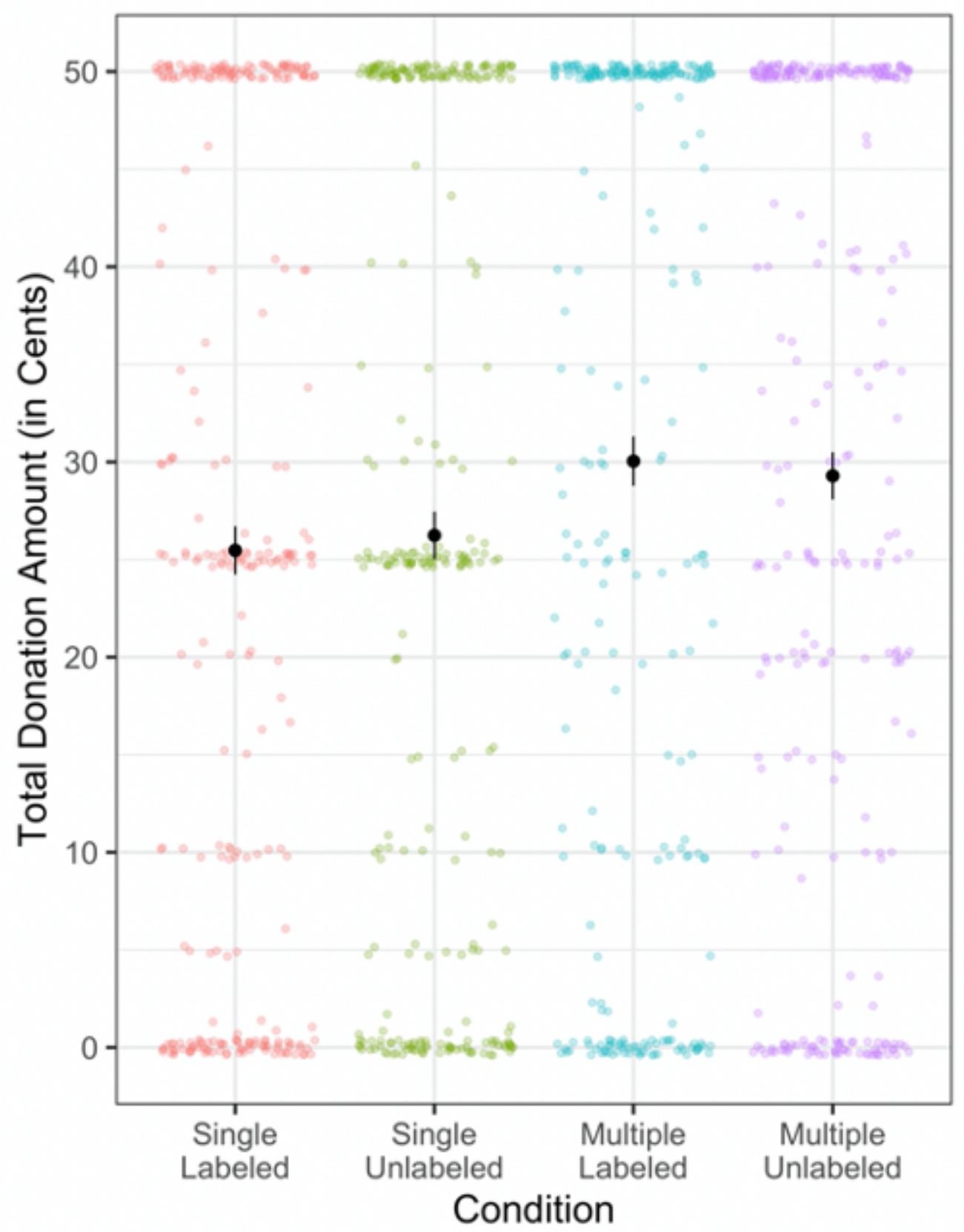

Figure 2

Total amount of money participants actually donated by condition in Experiment 2 Condition is displayed on the $x$-axis, donations in cents is displayed on the y axis. Error bars reflect $+/-1$ standard error. 
A)

Choose an amount:

NOTE: Your contribution will be divided evenly between

Entity 1 and Entity 2. Click here to allocate differently or view all recipients

\begin{tabular}{|l|l|l|l|}
\hline$\$ 25$ & $\$ 100$ & $\$ 250$ & $\$ 1000$ \\
\hline$\$$ & & \\
\hline
\end{tabular}

B)

Choose an amount:

Show simple form

Split Amount! $\$ 0$

Entity 1

Entity 2

$\$ 0.00$

\$ 0.00

Total

$\$ 0.00$

\section{Contribute}

D)

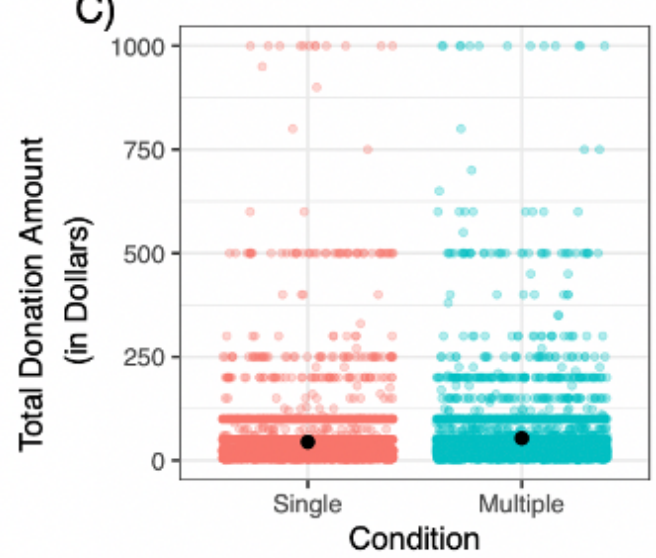

Contribute

\section{Figure 3}

TOP: Example of $(A)$ a single bid request and (B) a multiple bid request from Experiment 6 . Note that entity names are masked. BOTTOM: Total amount of money donated by condition (C) for all donations, (D) for donations $\$ 100$ and under, and (E) for donations $\$ 10$ and under. Each dot represents a donation. Condition is displayed on the $x$-axis, donations in dollars is displayed on the $y$ axis. Error bars reflect $+/-1$ standard error (note that they are difficult to see because they are very small).

\section{Supplementary Files}

This is a list of supplementary files associated with this preprint. Click to download.

- WeiszSMFINAL.docx 